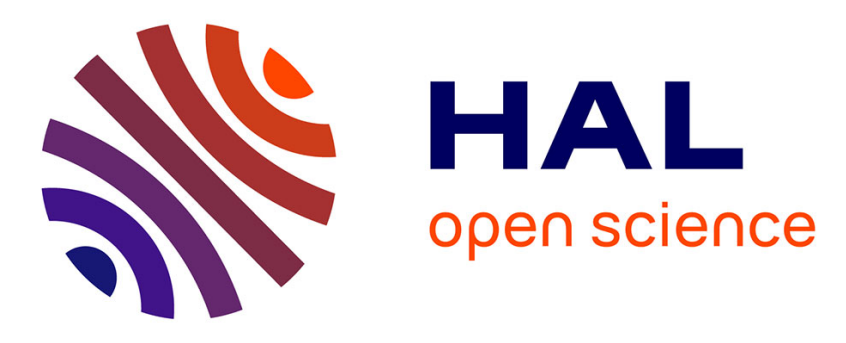

\title{
Data Management Requirements for PHM Implementation in SMEs
}

Nabil Omri, Zeina Al Masry, Sylvain Giampiccolo, N Mairot, Noureddine Zerhouni

\section{- To cite this version:}

Nabil Omri, Zeina Al Masry, Sylvain Giampiccolo, N Mairot, Noureddine Zerhouni. Data Management Requirements for PHM Implementation in SMEs. International Conference on Prognostics and Health Management (PHM 219), May 2019, Paris, France. 10.1109/PHM-Paris.2019.00046 . hal-03053029

\section{HAL Id: hal-03053029 https://hal.science/hal-03053029}

Submitted on 10 Dec 2020

HAL is a multi-disciplinary open access archive for the deposit and dissemination of scientific research documents, whether they are published or not. The documents may come from teaching and research institutions in France or abroad, or from public or private research centers.
L'archive ouverte pluridisciplinaire HAL, est destinée au dépôt et à la diffusion de documents scientifiques de niveau recherche, publiés ou non, émanant des établissements d'enseignement et de recherche français ou étrangers, des laboratoires publics ou privés. 


\title{
Data Management requirements for PHM implementation in SMEs
}

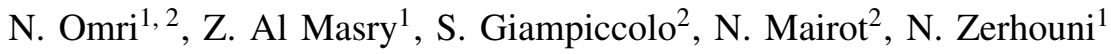 \\ ${ }^{1}$ FEMTO-ST institute, Univ. Bourgogne Franche-Comté, CNRS, ENSMM, \\ 24 rue Alain Savary, Besanon cedex, 25000, France \\ ${ }^{2}$ SCODER \\ 1 rue de la Forêt Z.A. l'Orée du Bois, Pirey 25480, France
}

\begin{abstract}
For industrial companies, product quality is a major factor in sustainability, cost optimization and revenue maximization. To achieve these objectives, companies aim to improve the reliability and capability of their manufacturing processes and thus improve the quality of their products. As a result, organizations are increasingly collecting data that describe their production systems in order to better control them using a Prognostics and Health Management (PHM) approach. However, the data collected are generally redundant and incomplete, which affects the results of the analysis. Many studies have been addressed this problem and proposed a data management module for the implementation of the PHM in the industrial domain. Recall that the characteristics of the data and their management depend on the collection area. Therefore, small and medium-sized enterprises (SMEs) are rarely studied, neither in data management nor in the implementation of PHM approaches. Existing works assume that SMEs can be treated in the same way as big corporation without taking into account the specifications and the needs of smaller businesses. In this paper, the specifications of SMEs are discussed in order to propose an adapted PHM process. This process is applied in a real case study and the first results are reported and discussed.
\end{abstract}

Index Terms-Small and Medium Enterprise (SME), Prognostic and Health Management (PHM), Data management, Data characterization.

\section{INTRODUCTION}

The zero-defect manufacturing is one of the key drivers of the company's performance. Thus, organizations are increasingly controlling their global process from the supplier to the final customer. This allows to identify the causes of the defects and react before that happens. In addition, industries aim to understand their process, minimize downtime and then maximize their production. In [1], Wang et al. affirm that the key factors to achieve stable production are monitoring the health state of an equipment and optimizing their decisionmaking. These goals cannot be achieved unless a good understanding of the production process which allows the system deviation identification. The latter information is taken into account when optimizing conjointly the maintenance and production planning. The used diagnosis and optimization methods gave birth to a new concept, which is the Prognostics and Health Management (PHM) [2].

Prognostics and Health Management is an approach that consists of studying the health state of an equipment and predicting its future evolution. This concept allows to control the production process and to implement suitable maintenance strategies [3]. In [4], authors affirm that PHM can be implemented using model based or data driven approaches. The first approach consists in building analytical models that are directly related to the physical processes which influence the health of the systems. Thus, a good comprehension of the physical process of components degradation and interaction is required. While the data driven approach consists in using historical monitoring data to model the evolution of the system until a failure occurs. In this case, the understanding of the physical process of the system is not necessary, but results depend on the quality of historical data. To deal with the problem of complex equipment for which operational data are not available, a new approach combining the benefits of model-based and data-driven methods is emerging. This technique is called the hybrid approach, which seems to provide more accurate results by reducing the uncertainty on the prediction [17]. The main idea of this concept is to use the physical process to describe the health state of the system and to control it using data-driven methods [4].

We here define PHM as a set of tools that can be used in cascade or separately to monitor the health state of a system, predict its future evolution and/or optimize decisions. These tools are implemented based on the historical data or the physical model of the system. Companies are gradually adopting the PHM concept to achieve their objectives. Thanks to the digitization, corporations are incrementally collecting data describing their production process. The collected data are then used to control and to improve the production process through some data driven PHM tools. This concerns usually big corporations such as the automotive field and electronics enterprises [16]. Up to our knowledge, the PHM tools are not yet very exploited in Small and Medium Enterprises (SMEs). 
The aim of this paper is to develop a first scheme of the implementation of PHM approach in SMEs. Our contribution is twofold. Firstly, we point out the difficulties of PHM practical use in SMEs and develop an adapted PHM approach for production process management. Secondly, we show the impact of quality data management on SMEs performance. This work is conducted in collaboration with SCODER enterprise which is a French SME specialized in the ultra-precise stamping for automotive application. The rest of this paper is organized as follows. Section II analyzes the data problems in SMEs. We then propose in Section III an adapted PHM approach for application in SME. Section IV describes the data management requirements in the case of SMEs. The results of a real case study are reported and discussed in Section $\mathrm{V}$ and finally a conclusion is presented in section VI.

\section{Problem Statement}

The increased amount of data in the industrial field requires appropriate treatment to meet the challenge of zero defect manufacturing [1]. In this context, data-driven PHM tools for industrial systems have attracted the attention of researchers and industrialists during the last decade [5]. Their works concern many fields such as the manufacturing, energy and transportation industries [16].

PHM approach can help manufacturers to better control their process and to reduce their total maintenance cost. As mentioned before, only big companies are using the latter approach. However, they need to collaborate with other IT companies to achieve their objectives [16]. As result, PHM for SME remains an unstudied domain, and even the few existing works are inspired from big corporations perspectives and have not taken into account the needs of SMEs. Many constraints were discussed in the literature and they are usually related to problems of data collection and analysis. In this work, we propose to classify these constraints into two main categories: resource-based constraints and organization-based constraints (see Figure 1).

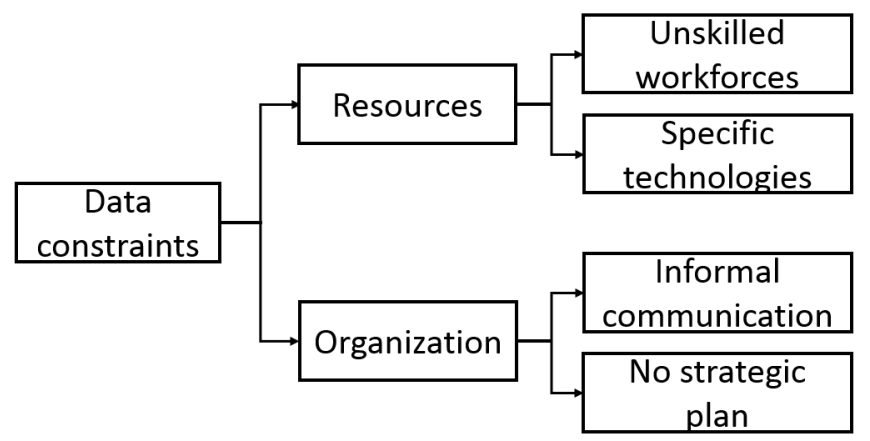

Fig. 1. Constraints of the development of data driven PHM tools for SMEs.

\section{A. Resource-based constraints}

Throughout the development of data-driven PHM tools, specific resources are requested, more particularly in the data collection and analysis steps. In this section, we only focus on human and technological resources. From a technological point of view, only 5\% of equipment in companies are digitally controlled [7]. This problem is mainly related to the lack of adequate measurement technologies to meet the needs of the manufacturing environment. In this context, SMEs can be considered as undeserved market [8].

For the human resources limits, authors in [10] affirm that unskilled workers join SMEs for a short period of time and when they are fully equipped with the required skills, they leave to join large enterprises. Consequently, advanced tasks such as data quality assessment and pre-processing cannot be supported by SMEs. To overcome this problem, some SMEs collaborate with external consulting firms to assist them to build their production history. But this collaboration call into question the profitability of the strategy.

\section{B. Organization-based constraints}

In this paragraph, we highlight the organizational factors. Usually, SMEs are characterized by a less formal organization and the communication between management and staff is close and informal [6]. Moreover, the information about the production process is limited in the know-how of operators without any documentation. In a case study of two SMEs, Boden et al. [11] reported that only very little documentation is available in these companies. Moreover, it never goes into details on how things are implemented. Also, information process is not digitized but it is based on documents edited by operators. For that purpose, the production history (if it exists) is created manually by workers. This process is one of the sources of data uncertainty [9]. In addition, SME owners are accused to be strategically myopic and lacking the long-term vision [18]. As result, medium and long-term strategies such as digitization and PHM implementation are rare in SMEs.

After identifying the limitations, it is then necessary to define a corresponded PHM approach that takes into consideration the problems presented above. The rest of the paper describes the chosen model along with its implementation. A case study is finally used to discuss the proposed roadmap.

\section{DATA DRIVEN PHM APPROACH FOR SMES}

In this section, we propose a new roadmap to implement data driven PHM tools in SMEs.

In [12], Vogl et al. propose a general flowchart of the process of PHM system development. This flowchart starts with a dependability and cost analysis in order to identify the most critical systems to control them. Then, a data management process is carried out. This process assembles the collection, processing, visualization and storing of data. When the measurement techniques are installed, a diagnostic and prognostic phase is conducted and the results are evaluated. Finally, workers are trained to use these new tools. In this flowchart, authors suppose that if there are problems in the 
testing phase, we need to check the used analysis methods and/or the data requirements. They also suppose that the measurement system is modified only as a consequence of the modification of the requirements. It is true that the used analysis methods have a great influence on the results but the data requirements are directly related to the objectives. So any change in these data requirements means that the objectives are also changed.

Our proposed roadmap is hence inspired from the above flowchart [12] and described in Figure 2. Therefore, the application mode in the new roadmap is adapted to the specifications of SMEs. The the different phases of our model are now presented.

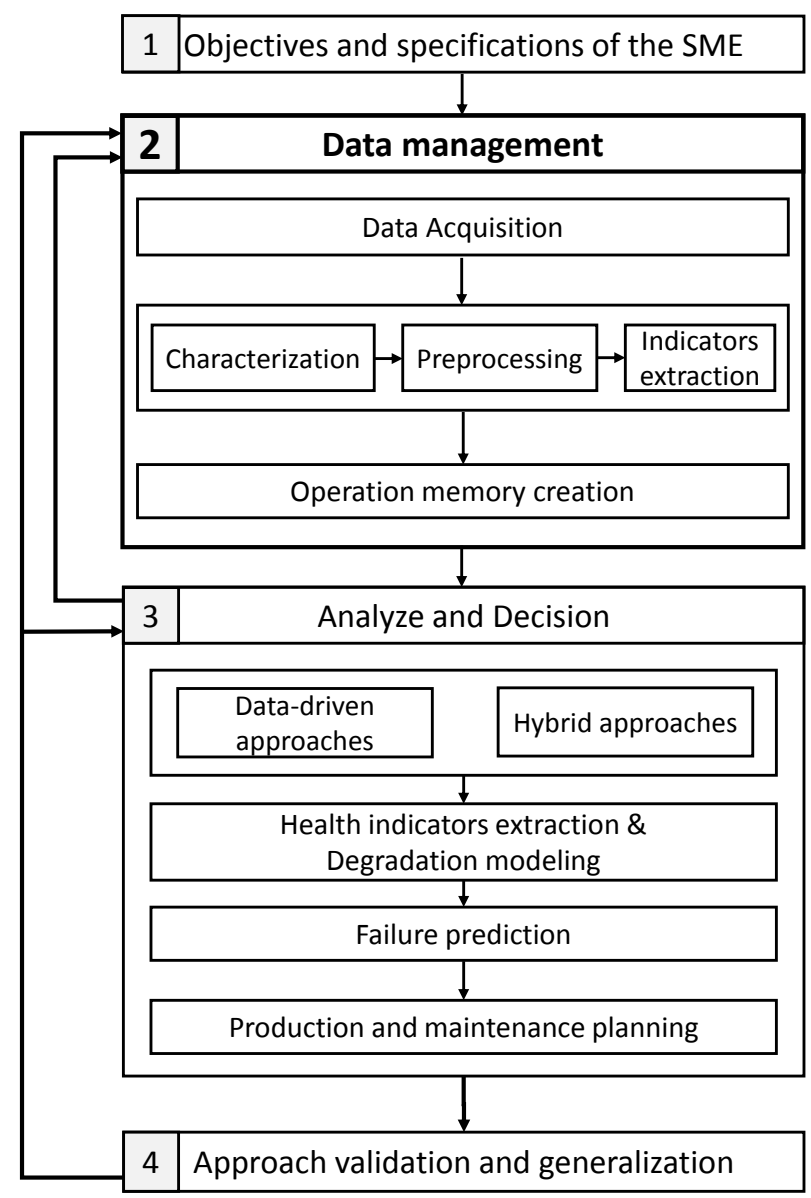

Fig. 2. The proposed roadmap.

\section{A. Objectives and specifications of the SME}

SMEs has many types of breakdowns related to different causes. This generates different type of costs. Since the available resources are limited, it is then difficult to focus on all these failures and repair them. Hence, Pareto analysis [13] and statistical analysis of downtime should be conducted to identify the most important downtime factors. According to the Pareto technique, $80 \%$ of breakdowns are related to only $20 \%$ of causes [14]. As a result, the first step in our roadmap is to use this technique to establish a list of components and equipment to be controlled. At the same time, this study must take into account the financial aspect and minimize the time of Return On Investment (ROI) of the strategy. This step is very important in the process, but the lack of data make it very difficult to achieve. To solve this problem, the know-how of the workers must be used and putted into formal/numeric documentation in order to store it for future applications.

\section{B. Data Management}

According to Vogl et al. [12], the data management step concerns data collection, processing, visualization and storage. In the case of SMEs, this step can go beyond these concepts to a more general notion which is the creation of the production memory. The creation of the production memory is a critical phase in this strategy. It hence need to be evaluated by some metrics. In [15], authors emphasize the strong link between data quality and adequacy of decisions and propose for that to characterize the collected data by metrics ensuring the desired objectives. The ISO/IEC 25024 standard [21] defines an exhaustive list of metrics that can describe data. These metrics are grouped into system dependent metrics and data inherent ones. According to the above, the process of production memory creation can be done in 4 steps:

Step 1. Data characterization according to the fixed objectives;

Step 2. Implementation of the measurement system that meet with the needed data quality;

Step 3. pre-processing of the collected data;

Step 4. Visualization and storage of the obtained information (production memory creation).

This phase consists in raw data collection and preprocessing in order to extract indicators about the health state and the performance of the system. Once the objectives are fixed, the characteristics of the required data can be defined. This concerns the required variables, their sources, frequency and period of measurement and the needed level of precision. Moreover, metrics must be identified to evaluate the adequacy between the measurement system and the fixed objectives. In this context, companies should capture the required data automatically and without human intervention [9] in order to guarantee an accepted level of precision.

However, in the case of SMEs, information declared by workers are very important to start the study even if their exactitude is not ensured. Usually, collected data present some problems such as noise, missing values, redundancy, etc [19]. For that purpose, a pre-processing module is used to treat these problems and improve the data quality. The pre-processing step is based on statistical methods and they mainly concerns data transformation, integration, cleaning and normalization [20]. The objectives of these techniques is to improve the description of the general state of the system. Moreover, the pre-processing phase allows to facilitate the visualization of the data, its understandability and storage. 


\section{Analysis and decision phase}

The main objective of this phase is to build decisions during a defined horizon in order to optimize the production and maximize the benefits. However, optimal decision cannot be taken without a good understanding of the system evolution.

Based on the collected data and extracted information, the analysis phase aims to build statistical and Artificial Intelligence models able to describe the health state of components and predict their breakdowns. The challenge is to treat a huge amount of data, one other challenge is to develop a suitable tools that can deal with data quality problem and achieve the fixed objectives. In fact, these tools must be able to give acceptable results even with small and noisy data. Based on existing works in the domain of PHM, we can find many analysis algorithm, it will be interesting to clarify the relation between data metrics and suitable analysis tool. The decision step aims to find an optimal planning of maintenance and production that allow to maximize the benefits. Decisions making is based on the the assimilation and interpretation of prognostics information [22].

However, there are a need to address the uncertainty behind the capitalized knowledge since their sources are different. Additionally, the dependency between the analysis and decision steps is not yet studied. For that purpose, it might be interesting to define some metrics for the decision phase and determine their relation with the uncertainty of prediction models build in the analysis phase.

\section{Validation and generalization}

The final phase of this roadmap is the validation of the developed tools and the training of the staff. To guarantee the success and the sustainability of the strategy, feed backs should be carefully analyzed in order to propose improvement actions. The iteratively improvement process must be continued in order to meet new challenges and achieve the objectives.

From an accessibility point of view, the developed tools must be well explained and documented. In addition, they should be presented as simple interfaces facilitating their use and allowing a smooth transfer of an understandable information.

\section{DATA MANAGEMENT REQUiREMENTS STRATEGY}

In line with the proposed roadmap in section III, in this section we will only focus on the data management step.

The data management process can be divided into three modules: Data collection, pre-processing and indicators extraction and visualization. The different steps of this process are shown in Figure 3.

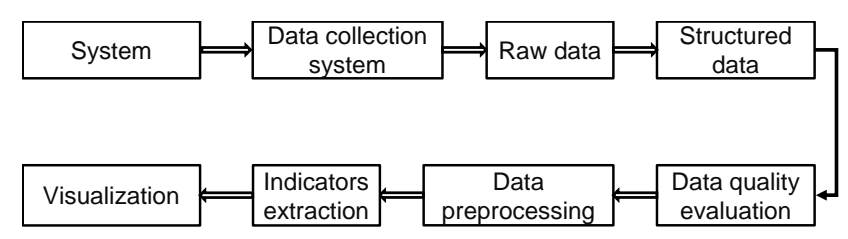

Fig. 3. The proposed data management approach.

\section{A. Characterization of SME data}

We first propose to characterize the SME data based on an empirical study conducted in SCODER. Literature reviews have been compared with the industrial reality and we found that some data problems are very common in SMEs. SCODER data has the characteristics of manual data, small volume of data, irrelevant and missing data.

- Manual data: Problems related to manual data collection can be found even in large companies. However, we have noted that this problem is more common among SMEs, which typically collect their data manually. The manual procedure leads to different description of the same event. Each worker has its own manner to represent the same information. Thus, in case of automatically data analysis, the manual data can be a major constraint for results accuracy.

- Small volume of data: The concept of data management is still not very used in the context of SMEs. As a result, SMEs are facing difficulties in valuing their data as a strategic resource. From the conducted empirical study, we notice that the collected data in SMEs are only considered to understand some critical problems. Once these problems are solved, the collected information are abandoned. In addition, storing data plays a crucial problem in SMEs since an advanced storage solutions are not available for production and maintenance data registration for a long period of time. The small volume of data hence remains insufficient to build accurate analysis models and particularly PHM approaches implementation.

- Irrelevant data: Since data management objectives are not usually known in SMEs, an exhaustive list variables to describe even a simple problem is collected. Consequently, the collected data may be irrelevant and redundant, which may affect the results or the decision to follow. In addition, this will generate a huge amount of unnecessary data, resulting in storage problems.

- Missing data: In the same context of manually collected data, workers consider that they loses a lot of time to record all the information concerning the production process. For that purpose, they are limited to only register the main events without going into details. Moreover, this data can be lost since it is saved on paper formats.

\section{B. Data pre-processing:}

Once the most common data problems in SMEs are identified, we come to study the most appropriate pre-processing technique for SMEs data quality improvement. 
- Data structuring: The data structure is the first step to consider in data pre-processing, which represents the way in which the data will be stored in the memory in a structured form. In the context of SMEs, data is stored and shared in different forms such as CSV, PDF and TXT files. To overcome this problem, we propose to structure all available data in a unified relational data file that easily allows subsequent data transformations.

- Data encoding: As mentioned above, available data in SMEs involve quantitative and qualitative information. The encoding aims to convert all the qualitative data into quantitative data in order to facilitate its pre-processing, analyze and storing. Moreover, the encoding allows unify the representation of data and subsequently facilitate its deployment. Based on our empirical study, we have noticed that many problems can be added to the converting of data such as non unified language message, different sensor code, etc. This problem will be more detailed in Section V.

- Data modeling: SME data could be irrelevant, noisy and presents some missing values. This can be explained by different facts such as errors caused by measurement system, analog-to-digital conversion and the manual recording. Filtering tools will be deployed to solve noisy issues. To deal with the problem of irrelevant or redundant variables, features selection techniques are very used to select the relevant variables for solving an analysis problem.

- Data generation: In the case of small volumes of data, generating them can be an efficient solution. Statistical tools can be used to create similar data that can be used to train diagnosis and prognosis algorithms.

\section{Performance indicator extraction}

In order to assess the performance of SMEs, a set of Performance Indicators (PIs) must be defined. These indicators are used to identify critical problems to handle by SMEs and to evaluate the effectiveness of the proposed PHM approach.

Organizations need to assess their systems availability, operational efficiency and their quality rate [25]. The aggregation of all these indicators can be explained as an unified indicator which is the Overall Equipment Effectiveness (OEE). The $\mathrm{OEE}$ is one of the most cited indicators in the literature and the most implemented by the companies to evaluate the efficiency of their machines [26]. In [23], authors affirms that OEE is not only an operational measure but also an indicator of process improvement activities. To sum up, the OEE is an essential quantitative metric for measuring productivity in manufacturing operations and is given by

$$
O E E=A v \times O E \times Q R
$$

where $A v$ refers to the ratio of time in which the machine is really available and the time that it is supposed to be available; $O E$ describes the proportion of time in which machine produce parts out of available and $Q R$ is the percentage of conforming parts from the total production.

The different indicators needed for OEE computation describe the losses in terms of production time for an equipment. Usually, a planned production time (PPT) is fixed for which the machine is considered in a production mode. Nevertheless, the spontaneous stops will modify this time into an actual production time (APT). In addition, the efficiency loss will reduce the APT into a net production time (NPT), in which the machine produce parts. After elimination of the non-conforming parts, we get the time of conforming parts production which is called the valuable production time (VPT). Figure 4 displays the above information.

\begin{tabular}{|c|c|}
\hline \multicolumn{2}{|c|}{ Planned Production Time } \\
\hline Actual Production Time & Unplanned stops \\
\hline Net Production Time & Loss of productivity \\
\hline Valuable Production Time Loss of quality & \\
\hline
\end{tabular}

Fig. 4. Different types of losses on an equipment (inspired from [24]).

Finally, the indicator are computed as follows

$$
\begin{aligned}
A v & =\frac{A P T}{P P T} ; \\
O E & =\frac{N P T}{A P T} ; \\
Q R & =\frac{V P T}{N P T} .
\end{aligned}
$$

\section{CASE STUdy AND Discussion}

We here consider a mechanical press which works on cutting, bending, forming and laminating of metal parts for automotive applications. For production data collection, an integrated measuring system is already implemented. Our proposed approach, particularly the data management step, is now applied.

The used data are collected from November 2017 to October 2018 and recorded in 53 CSV files. The amount of generated data is about 235 Mo. Each data raw consists of many variables such the occurrences of failures, their causes and the start and end times of failures. However, some information could be redundant or irrelevant. We hence start by the conversion of the CSV files into a relational database to facilitate the manipulation of the data and more particularly to identify the problems.

Figure 5 shows the structure of the developed relational database. We have pointed out that some failure messages are recorded with different languages and many sensors are represented in different codes.

Figure 6 shows some examples of these problems that were captured from the original CVS files. To be more precisely, some sensors messages are recorded in different 


\begin{tabular}{|l|l|l|}
\hline \multicolumn{1}{|c|}{ Failure } \\
\hline $\begin{array}{l}\text { Failure_Id } \\
\text { Cause_Id } \\
\text { Date } \\
\text { Start_Time } \\
\begin{array}{l}\text { End_Time } \\
\text { Counter }\end{array}\end{array}$ \\
\hline
\end{tabular}

Fig. 5. The database structure.

languages (French and German). By studying this problem, we found that it concerns the measuring system where the message language can be modified during interventions on the machine. Moreover, the references of some sensors are represented by different codes. This can be easily solved in the case of few sensors which is not the case here, where we have an important number of sensors and amount of data to treat. Consequently, these problems make the automatic data processing complicated.

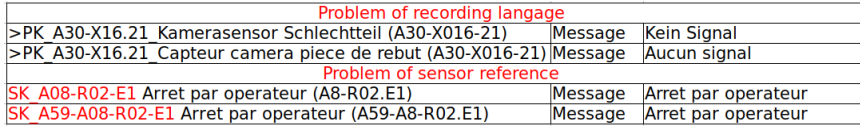

Fig. 6. Example of problems related to the data recording.

The next step is to remove the redundant information while optimizing the volume of our data. For example, the breakdown time is removed since it can be obtained from the start and end times. In addition, the text and symbolic information such as the sensor's codes are encoded in numeric representation. This transformation allows to unify the information representation and reduce their volume. In fact, text information occupies memory space more than a numeric representation. We can now extract some indicators that allows to measures the performance of the mechanical press. Using the information about the breakdowns and their causes, these latter are classified into planned breakdowns and unscheduled stops. Once the consequent time for each breakdown type is calculated, the OEE indicator is computed through different methods. This allows to evaluate the importance of data management within the company. In Table I, we display the values of OEE indicator computed manually in SCODER, using the raw data and using the pre-processed data. As we can seen, the results are more or less different. For the raw data and pre-processed data, the OEE values are almost similar but lower than the manual one as expected. This can be explained by the fact that almost all the breakdowns are totally recorded. However, the $A v$ and the $O E$ are different even between raw data and pre-processed data and this is due to the language problem mentioned above. Consequently, the classification of these failures into planned breakdowns and unscheduled stops is wrongly done. When manually recording data, operators record only the principal failures without considering the micro failures which could lead to important failures and productivity losses.
TABLE I

EFFICIENCY INDICATORS.

\begin{tabular}{|l|l|l|l|l|}
\hline Indicators & Av & OE & QR & OEE \\
\hline Raw data & $84.7 \%$ & $81.9 \%$ & $99.9 \%$ & $69.3 \%$ \\
\hline Pre-processed data & $\mathbf{8 6} \%$ & $\mathbf{8 0 . 8} \%$ & $\mathbf{9 9 . 9} \%$ & $\mathbf{6 9 . 4} \%$ \\
\hline Manual data & $87.2 \%$ & $95.8 \%$ & $99.9 \%$ & $83.5 \%$ \\
\hline
\end{tabular}

Additionally, we notice that operational efficiency should be improved. For that purpose, we started to analyze the small breakdown causes. Figure 7 shows the occurrence of these breakdowns as a function of their total repairing time. We can remark that only two types of breakdown are responsible of $40 \%$ of the total repairing time and the breakdowns caused by operators represent $23 \%$ of all the breakdowns time. Future works will be dedicated to study in details these failures and reduce their occurrences.

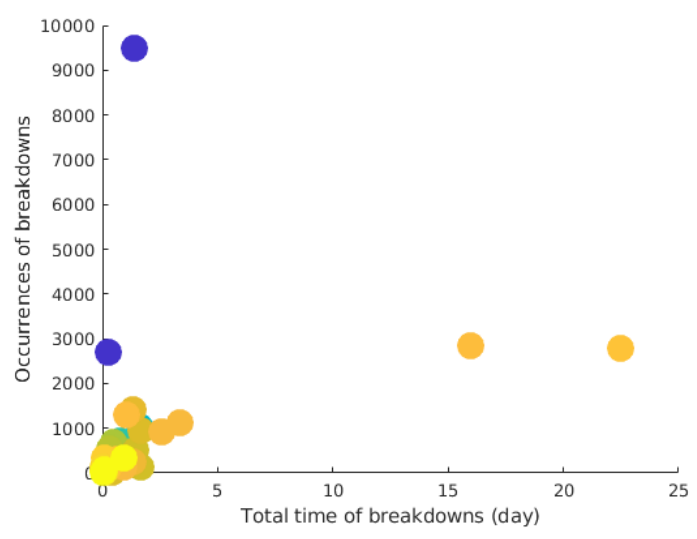

Fig. 7. Breakdowns and their total repairing time.

To sum up, this work is the first step of a project of PHM development and implementation in SCODER. It seems that SCODER, and SMEs in general, have particular problems with data collection (mostly manually) which affects the overall company analysis. Moreover, unnecessary data collection in SMEs leads to a storage problem. Adequate frequency collection and data volume are two important issues to look for. This will be studied in future work in order to establish a data management guide for SMEs.

\section{CONCLUSION}

This paper deals with the data management problems in SMEs in order to develop an adapted data driven PHM tools for this kind of organizations. The proposed approach was applied to a real case study. This topic is still under development for both data management requirements and PHM implementation for SMEs. Our work were limited to the data management step and the analysis and decision phases are not yet studied.

This can be seen as an initiation to future work. It would be interesting to more focus on the data quality models and propose a numeric indicator that characterize the data and 
relate them to the main objective. Also, one could think about advanced pre-processing of data in order to improve their quality and make them useful for decision purposes.

\section{REFERENCES}

[1] K. S. Wang. "Towards zero-defect manufacturing (ZDM)a data mining approach." Advances in Manufacturing 1.1 (2013): 62-74.

[2] L. JAY, ET AL. Prognostics and health management design for rotary machinery systemsReviews, methodology and applications. Mechanical systems and signal processing 42.1-2, 2014, pp. 314-334.

[3] P. Michael. Prognostics and health management of electronics. Encyclopedia of Structural Health Monitoring(2009).

[4] P. Michael. A prognostics and health management roadmap for information and electronics rich systems. IEICE ESS Fundamentals Review 3.4, 2010, pp. 4-25, 4-32.

[5] M. A Kouali, ET AL. Industrial Internet of Things-based Prognostic Health Management: A Mean-Field Stochastic Game Approach. IEEE Access (2018).

[6] D. Susanne and G. Bruns. Knowledge Management in Small and Medium-Sized Enterprises. The Palgrave Handbook of Knowledge Management. Palgrave Macmillan, Cham, 2018. 495- 514

[7] P. Waurzyniak. Why manufacturing needs real-time data collection. Manufacturing Engineering (2015): 53- 61

[8] M. Helu, ET AL. Identifying performance assurance challenges for smart manufacturing. Manufacturing letters 6 (2015): 1-4.

[9] S. Aleksei, et al. Real time production monitoring system in SME/Reaalajas toimiv tootmise seiresusteem. Estonian Journal of Engineering 19.1 (2013): 62-76

[10] S. M. Bala. Innovation and growth of engineering SMEs in Bangalore: Why do only some innovate and only some grow faster? Journal of Engineering and Technology Management 36 (2015): 24- 40.

[11] B. A. Oden, et al. Knowledge sharing practices and the impact of cultural factors: reflections on two case studies of offshoring in SME. Journal of software: Evolution and Process 24.2 (2012): 139-152.

[12] G. W. Vogl, B. A. Weiss, and M. A. Donmez. Standards for prognostics and health management (PHM) techniques within manufacturing operations. National Institute of Standards and Technology Gaithersburg United States, 2014.

[13] R. A. Hall, P. F. Knights, and L. K. Daneshmend. Pareto analysis and condition-based maintenance of underground mining equipment. Mining Technology 109.1 (2000): 14-22.

[14] CML. Rahman. Assessment of total productive maintenance implementation in a semi automated manufacturing company through downtime and mean downtime analysis. Industrial engineering and operations management (IEOM), 2015 international conference on. IEEE, 2015.

[15] K. Iris et al. Metrics for the evaluation of data quality of signal data in industrial processes. Industrial Informatics (INDIN), 2017 IEEE 15th International Conference on. IEEE, 2017.

[16] K. Daeil, et al. IoT-based prognostics and systems health management for industrial applications. IEEE Access 4 (2016): 3659-3670.

[17] P. Pillai, A. Kaushik, S. Bhavikatti, A. Roy, and V. Kumar. A Hybrid Approach for Fusing Physics and Data for Failure Prediction. International Journal of Prognostics and Health Management (2016): 7(025), $1-12$.

[18] C. Wang., E. Walker, J. Redmond. Explaining the lack of strategic planning in SMEs: The importance of owner motivation. 2007

[19] S. R. Gallego, et al. A survey on data pre-processing for data stream mining: Current status and future directions. Neurocomputing 239 (2017): 39- 57.

[20] S. Garcia, et al. Big data pre-processing: methods and prospects. Big Data Analytics 1.1 (2016): 9.

[21] ISO/IEC 25024, Software engineering - software product quality requirements and evaluation (square) - measurement of data quality. 2015.

[22] I. Naresh, K. Goebel and P. Bonissone. Framework for post-prognostic decision support. Aerospace Conference, 2006 IEEE. IEEE, 2006.

[23] B. Dal, P. Tugwell, and R. Greatbanks, Overall equipment effectiveness as a measure of operational improvement, Int. J. Operations Prod. Manage., vol. 20, no. 12, pp. 14881502, 2000

[24] N. Seiichi. "Introduction to TPM: Total Productive Maintenance (preventative maintenance series)." Hardcover. ISBN 0-91529-923-2 (1988).

[25] A. J. De Ron, and J. E. Rooda. "OEE and equipment effectiveness: an evaluation.” International Journal of Production Research 44.23 (2006): 4987-5003.
[26] M. Peter, and L. Pintelon. "Performance measurement using overall equipment effectiveness (OEE): literature review and practical application discussion." International journal of production research 46.13 (2008): 3517-3535 